\title{
Maternal sleep-disordered breathing and the risk of delivering small for gestational age infants: a prospective cohort study
}

\author{
Sushmita Pamidi, ${ }^{1}$ Isabelle Marc ${ }^{2}$ Gabrielle Simoneau, ${ }^{3,4}$ Lorraine Lavigne, ${ }^{1}$ \\ Allen Olha, ${ }_{1}^{1}$ Andrea Benedettii, ${ }^{3,4}$ Frédéric Sériès, ${ }^{5}$ William Fraser, ${ }^{6}$ \\ François Audibert, ${ }^{6}$ Emmanuel Bujold, ${ }^{2}$ Robert Gagnon, ${ }^{7}$ Kevin Schwartzman, 1,3,4 \\ R John Kimoff ${ }^{1}$
}

\begin{abstract}
${ }^{1}$ Respiratory Division and Sleep Laboratory, McGill University Health Centre, Montreal, Quebec, Canada

${ }^{2}$ Departement de Pédiatrie, Centre Hospitalier de I'Université Laval, Québec, Québec, Canada

${ }^{3}$ Respiratory Epidemiology and Clinical Research Unit, McGill University Health Centre, Montreal, Quebec, Canada ${ }^{4}$ Department of Epidemiology, Biostatistics \& Occupational Health, McGill University Health Centre, Montreal, Quebec, Canada

${ }^{5}$ Departement de Pneumology, Institut de Cardiologie et de Pneumologie de Québec, Université Laval, Québec, Quebec, Canada

${ }^{6}$ Hôpital Ste-Justine, Centre de recherche, Université de Montréal, Montreal, Quebec, Canada

${ }^{7}$ Department of Obstetrics and Gynecology, McGill University Health Centre, Montreal, Quebec, Canada
\end{abstract}

\section{Correspondence to} Dr Sushmita Pamidi, Respiratory Division, McGill University Health Centre-Glen Site, Block D-D05.2506 1001 Decarie Blvd, Montreal, Quebec, Canada H4A 3J1; sushmita.pamidi@mcgill.ca

Received 6 November 2015 Revised 9 February 2016 Accepted 11 March 2016 Published Online First 15 April 2016 CrossMark 2016;71:719-725.

\section{ABSTRACT}

Objective It is unclear whether objectively measured maternal sleep-disordered breathing (SDB) leads to poor fetal outcomes. In this study, we prospectively assessed whether polysomnography-based diagnosis of SDB in the third trimester is associated with the delivery of small for gestational age (SGA) infants.

Study design Participants were recruited from a multicentre pregnancy cohort study. Eligible participants were evaluated for SDB based on symptoms (snoring and/or witnessed apnoeas assessed using the Pittsburgh Sleep Quality Index questionnaire) and in-home complete polysomnography in the third trimester. SGA was defined as <10th centile using customised birthweight centiles adjusted for maternal parity, prepregnancy body mass index (BMI), ethnicity, gestational age and infant sex. Results Of the 234 pregnant participants who completed a sleep study, $82 \%$ were Caucasian, with mean (SD) age of 31 (4.3) years and a prepregnancy BMI of $23(4) \mathrm{kg} / \mathrm{m}^{2}$. The delivery of SGA infants occurred in $27(12 \%)$ of the study participants. The symptoms of SDB had poor overall sensitivity and specificity for diagnosing SDB identified by polysomnography. Symptoms of SDB in the third trimester demonstrated a potential association with delivering an SGA infant, however this did not reach statistical significance (OR $2.36(95 \% \mathrm{Cl} 0.85$ to 6.54 , $\mathrm{p}=0.10)$ ). However, the odds of delivering an SGA infant were significantly increased with polysomnography-based diagnosis of maternal SDB (using apnoea-hypopnoea index cut-off of 10 , OR $2.65(95 \% \mathrm{Cl} 1.15$ to 6.10 , $\mathrm{p}=0.02)$ ).

Conclusions Objectively measured SDB in the third trimester is significantly associated with the delivery of SGA infants.

\section{INTRODUCTION}

Infants with intrauterine growth restriction (IUGR) that are born small for gestational age (SGA) are at risk for greater perinatal morbidity and mortality. ${ }^{1}$ Later in life, being born SGA may be a risk factor for developing cardiometabolic complications and neurological disabilities. ${ }^{2}$ IUGR may be caused by a variety of maternal, placental and/or fetal factors. In many cases, the exact aetiology is not identified and management is limited to close surveillance and early

\section{Key messages}

What is the key question?

- Does maternal sleep-disordered breathing confer an increased risk of delivering babies that are small for gestational age?

\section{What is the bottom line?}

- In a cohort of relatively healthy and non-obese women, we report an increased risk of delivering small for gestational age infants with maternal sleep-disordered breathing determined by polysomnography.

\section{Why read on?}

- Demonstration of a link between fetal outcomes and maternal sleep-disordered breathing suggest that future clinical trials are needed to investigate whether treatment of sleep-disordered breathing in pregnancy improves the lifelong health of the newborn infant.

delivery. ${ }^{4}$ The pathophysiology of IUGR is characterised by insufficient uteroplacental blood flow due to vasoconstriction. ${ }^{5}{ }^{6}$ Given the limited treatment options of IUGR, the identification of reversible pathogenic factors may improve fetal outcomes.

Sleep-disordered breathing (SDB) is characterised by repetitive partial or complete upper airway obstructions during sleep, resulting in sleep fragmentation and/or recurrent hypoxia-reoxygenation. Multiple downstream effects of SDB, such as haemodynamic fluctuations, increased sympathetic activity, oxidative stress, endothelial dysfunction and systemic inflammation ${ }^{78}$ have been associated with adverse cardiac and vascular sequelae in non-pregnant populations. ${ }^{9-12}$ Animal studies have demonstrated that maternal intermittent hypoxia, a model for SDB, leads to low birth weight. ${ }^{13}{ }^{14}$ Given the known adverse vascular consequences of untreated SDB in the general population, it is plausible that SDB during pregnancy may contribute to altered haemodynamics and diminished placental tissue perfusion, with a potential to decrease fetal growth.

A growing number of human studies indicate that symptoms of SDB increase and progress 
throughout pregnancy, with $14-35 \%$ of pregnant women reporting habitual snoring in the third trimester. ${ }^{15-17}$ Mechanisms of SDB are likely multifactorial and include weight gain, fluid shifts ${ }^{18}$ and rhinitis. ${ }^{19}{ }^{20} \mathrm{~A}$ recent meta-analysis demonstrated that maternal SDB is associated with an increased risk of delivering low birthweight infants (pooled OR 1.39, 95\% CI 1.14 to 1.65$).{ }^{21}$ However only two out of eight studies relied on objective, rather than subjective measures of sleep apnoea. Moreover, these two studies ${ }^{22} 23$ were retrospective cohort and database linkage studies. Prospective cohort studies assessing this association are therefore required. There is effective treatment for SDB (continuous positive airway pressure; CPAP), which could therefore potentially be a novel antenatal therapeutic option for preventing SGA.

The objective was to perform a prospective cohort study assessing the association between maternal SDB and infants born SGA using complete in-home (level II) polysomnography (PSG) in the third trimester. Preliminary results of this study have been previously reported in the form of abstracts. ${ }^{24} 25$

\section{METHODS}

\section{Study participants}

Participants for the SGA and maternal SDB substudy were recruited between September 2011 and March 2013 from a larger pregnancy cohort (3D Birth Cohort of the Integrated Research Network in Perinatology of Quebec and Eastern Ontario; IRNPQEO) aimed at examining determinants of various maternal and fetal outcomes. For the SGA and maternal SDB substudy, the inclusion criteria were a third trimester ultrasound that showed $<75$ th centile predicted birth weight ${ }^{26}$ in order to minimise the number of large for gestational age babies (>90th centile birth weight) in our cohort (this criteria was applied to all participants, irrespective of SDB risk), a singleton pregnancy and no current treatment for a known sleep disorder. Study coordinators of the parent cohort approached eligible participants for participation in a research substudy on sleep in pregnancy.

\section{Study design}

In this prospective cohort study, eligible and interested participants were recruited in the third trimester and underwent a level II home sleep study soon after recruitment. The primary measure of exposure status (ie, presence or absence of SDB) was ascertained by PSG performed during the third trimester of pregnancy, since SDB is known to significantly improve after delivery. $^{27}$ The study was approved at all participating institutions through the Quebec Ministry of Health Multi-Centre Research Ethics process (for which the primary reviewing site was Ste-Justine Hospital, coordinating centre for the IRNPQEO cohort study).

\section{General cohort and outcome assessments}

As part of the parent cohort study measurements, all subjects were evaluated at 10-14 weeks, 20-24 weeks and 32-35 weeks gestational age. Maternal characteristics including ethnicity, marital status, education, prepregnancy weight, smoking exposure history, and medical and obstetric history were obtained through questionnaires and chart review. A positive smoking history was defined as smoking at least an average five cigarettes/day during pregnancy. Small for gestational age was defined as a birth weight $<10$ th centile using customised birth centile curves with a US population reference standard. ${ }^{28} 29$ The customised birth centiles are adjusted for infant sex, gestational age at birth and maternal constitutional factors that have been consistently found to be predictive of fetal growth ${ }^{28} 29$ including maternal ethnicity, parity, prepregnancy weight and height. This adjustment is made accessible through an online customised individual growth chart calculator called GROW ${ }^{30}$ (Gestational Related Optimal Weight; gestation.net) developed by Gardosi et $a l^{28} 29$ to calculate the individual customised optimal birth weight based on the aforementioned maternal and fetal characteristics, and thus allows comparison of an actual infant birth weight against its own predicted weight or optimal growth potential.

\section{Polysomnography}

One night of in-home, complete overnight PSG (Titanium, Embla, Natus Medical, San Carlos, California, USA) was performed during the late third trimester, after the third trimester ultrasound. A sleep technologist installed the recording equipment in the participant's home on the evening of the study to verify signal quality. Scorers were blinded to the ultrasound results. Sleep-wake state, arousals and periodic limb movements were scored according to current American Academy of Sleep Medicine (AASM) criteria. $^{31}$ Respiratory events were scored using standard Chicago criteria, ${ }^{32}$ which are more sensitive than current AASM criteria. ${ }^{33}$

\section{Sleep questionnaire data}

The Pittsburgh Sleep Quality Index (PSQI) ${ }^{34}$ was administered during the routine first and third trimester study visits. The PSQI consists of 19 self-rated questions regarding sleep quality and 5 questions that are targeted to the bed partner of the participant (if applicable). These five questions include two questions that relate to symptoms of sleep apnoea: 'loud snoring' and 'long pauses between breaths while asleep'. Classification as to snoring or witnessed apnoeas was based simply on the presence or absence of these reported symptoms.

\section{Statistical analysis}

For sample size calculation, the prevalence of SDB in pregnant women is unknown. Since up to $35 \%$ of women present with symptoms of SDB in the third trimester, ${ }^{15}$ and anticipating the possibility of participation bias, we felt the rate of exposure could be similar to the rate of non-exposure depending on the threshold of the apnoea-hypopnoea index (AHI) used. Using a prevalence of SGA of $6 \%$, among non-snorers ${ }^{35}$ we estimated that to detect an OR of $\sim 2.5$ (based on previous symptom-based studies $^{16}{ }^{35}$ ) of SGA among SDB versus non-SDB with a power of $80 \%(\beta 0.2)$ and $\alpha 0.05$, we would require a total sample size of $\sim 225$ subjects. Means with SDs were used for descriptive statistics for continuous variables and numbers and proportions for categorical variables. Univariate logistic regression analysis was used to determine the association between SDB (using various threshold values for AHI) and other sleep parameters and delivery of an SGA infant. Logistic or linear regression modelling (depending on the outcome of interest) was used to calculate $p$ values for trend (Cochrane-Armitage trend test ${ }^{36}$ ), when comparing outcome values across categories of increasing severity of AHI. An ordinal variable for AHI was created by using the median AHI of each category and the $\mathrm{p}$ value associated with this was used as the $\mathrm{p}$ value for trend. Comparisons of mean AHI, oxygen desaturation index, obstructive hypopnoea index, hypopnoea arousal index for SGA and non-SGA babies used the Student's t test. Statistical analyses were performed using JMP, V.11 (SAS Institute, Cary, North Carolina, USA, 1989-2007) and R (RStudio V.0.99.441). 


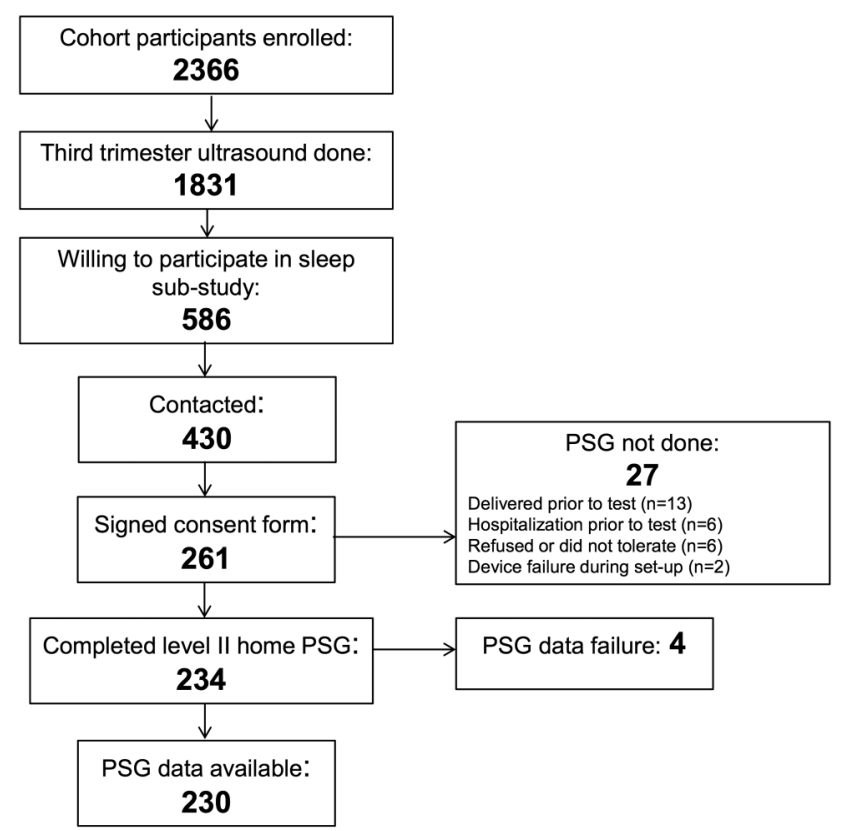

Figure 1 Participant flow diagram. The number of participants who were enrolled, consented for the study and completed a level II polysomnogram. PSG, polysomnography.

\section{RESULTS Study participants}

The participant flow diagram for recruitment and enrolment from the parent IRNPQEO cohort is shown in figure 1. Of 261 women who consented for participation in the substudy, $10 \%$ did not have a PSG for various reasons. Thus, 234 women from the parent cohort participated in the sleep substudy and completed an at-home level II PSG during the third trimester. Baseline participant characteristics are shown in table 1. Participants in the sleep substudy were predominantly non-obese (body mass index (BMI) $<30 \mathrm{~kg} / \mathrm{m}^{2}$ ) and Caucasian (table 1), with 6\% Latin American, 3\% Arabic or West Asian, 3\% African American and 2\% Native American. Questionnaire data revealed that a small minority smoked during pregnancy (table 1), three reported depression and none had pre-existing diabetes or hypertension. The prevalence of gestational hypertension was $3 \%(n=7)$ while gestational diabetes was reported in $7 \%$ $(n=17)$ of pregnancies. Active asthma was reported in $9 \%$ $(n=18)$, and only 1 participant reported active cardiovascular disease. With increasing AHI, there was a significant increase in maternal age, BMI and neck circumference (table 1).

\section{Symptom and PSG-based evaluation of SDB}

In the first trimester, 182 participants completed the PSQI and $54(31 \%)$ reported any degree of snoring and/or witnessed apnoeas in the first trimester. This increased significantly to 87 $(51 \%)$ by the third trimester $(\mathrm{p}<0.0001)$. There was a $35 \%$ incidence of new symptoms of SDB from the first trimester to the third trimester.

The third trimester PSG was performed at a mean of 36.6 $( \pm 1.4)$ weeks of gestational age. After six technical failures $(3 \%$ rate), PSG data were available on 230 participants (figure 1). Since AHI cut-offs for defining SDB are not well established in pregnancy, table 1 displays demographic characteristics of the cohort as divided by severity of SDB based on various AHI categories. Using AHI cut-offs of 5 events/h, 10 events/h, 15 events/h and 30 events/h, the prevalence of SDB in this cohort was $66 \%, 30 \%, 16 \%$ and $2.6 \%$, respectively. Table 2 demonstrates PSG sleep variables. There was a trend towards decreased PSG total sleep time and increased N1 sleep as the severity of AHI categories increased. In addition, there was significantly reduced N3 sleep but no change in rapid eye movement (REM) sleep as SDB severity increased. The mean AHI of the cohort was $9.1 \pm 8.2$ events $/ \mathrm{h}$. As shown in table 2, SDB in the third trimester of pregnancy was predominantly characterised by obstructive hypopnoeas associated with microarousals, rather than apnoeas or events associated with oxygen desaturation.

The proportion of participants who reported snoring and/or witnessed apnoeas in the first trimester and third trimester significantly increased with severity of AHI category (figure 2). However, the sensitivity and specificity for snoring and/or witnessed apnoeas to predict maternal SDB in the third trimester, irrespective of AHI cut-off, was poor overall (table 3).

\section{Association between symptom-based and PSG-based diagnosis of SDB and delivery of SGA infants}

The total number of SGA deliveries in this cohort was $27(12 \%)$. Gestational age and birthweight data are shown in table 4 . The rate of preterm birth as defined by a gestational age at delivery of $<37$ weeks did not differ across AHI categories. While absolute birth weight did not differ across AHI categories (table 4), figure 3 demonstrates an increased proportion of SGA births based on customised birthweight centile born to mothers with increasing SDB severity. Furthermore, as shown in figure 4, there were significantly higher mean values for key measures of SDB severity among the mothers of infants born SGA compared with non-SGA.

In addition, almost all PSG variables, including SDB defined by various $\mathrm{AHI}$ thresholds (5, 10 and 15), and continuous PSG

Table 1 Demographic and baseline clinical characteristics of the pregnancy cohort according to the AHI stratum

\begin{tabular}{|c|c|c|c|c|c|}
\hline & Complete cohort $(n=234)$ & AHI $<5 \quad(n=77)$ & $5 \leq \mathrm{AHI}<10(\mathrm{n}=84)$ & $10 \leq$ AHI $<15(n=33)$ & $A H I \geq 15(n=36)$ \\
\hline Age, years & $31.0(4.3)$ & $29.6(4.1)$ & $31.3(4.4)$ & $32.1(4.5)$ & 32.7 (3.7) \\
\hline Caucasian ethnicity & $190(82 \%)$ & $64(83 \%)$ & $72(86 \%)$ & $24(75 \%)$ & $27(75 \%)$ \\
\hline Number of years in school & $18.0(9.3)$ & $18.4(12.1)$ & $17.6(3.0)$ & $17.0(4.1)$ & $18.9(14.2)$ \\
\hline Nulliparous & $99(42 \%)$ & $38(49 \%)$ & $33(39 \%)$ & $13(41 \%)$ & $14(39 \%)$ \\
\hline BMI prepregnancy $\left(\mathrm{kg} / \mathrm{m}^{2}\right)$ & $22.8(3.8)$ & $22.0(3.3)$ & $22.4(3.6)$ & $23.0(3.5)$ & $25.2(4.6)$ \\
\hline $\mathrm{BMI} \geq 30 \mathrm{~kg} / \mathrm{m}^{2}$ & $16(7 \%)$ & $2(3 \%)$ & $6(7 \%)$ & $2(7 \%)$ & $6(17 \%)$ \\
\hline Neck circumference (cm) & $33.3(2.0)$ & $33.0(1.8)$ & $33.0(1.9)$ & $33.7(2.3)$ & $34.6(1.6)$ \\
\hline Smoking during pregnancy & $15(6 \%)$ & $4(5 \%)$ & $6(7 \%)$ & $3(9 \%)$ & $2(6 \%)$ \\
\hline
\end{tabular}

Values are means (SD) or numbers (\%).

p Values represent statistical tests by analysis of variance (ANOVA) for continuous variables and Fisher's exact test for non-parametrical and $\chi^{2}$ test for parametrical categorical variables. The neck circumference was taken at the time of the third trimester polysomnogram. Smoking during pregnancy was defined as smoking at least five cigarettes/day. $\mathrm{AHI}$, apnoea-hypopnoea index; BMI, body mass index. 
Table 2 Polysomnographic sleep characteristics according to apnoea-hypopnoea index stratum

\begin{tabular}{|c|c|c|c|c|c|}
\hline & Complete cohort $(n=234)$ & $A H I<5(n=77)$ & $5 \leq \mathrm{AHI}<10(\mathrm{n}=84)$ & $10 \leq A H I<15(n=33)$ & $A H I \geq 15(n=36)$ \\
\hline PSG total sleep time (h) & $6.7(1.4)$ & $6.7(1.6)$ & $6.9(1.1)$ & $6.5(1.4)$ & $6.2(1.6)$ \\
\hline N1 sleep (min) & $43.4(21.1)$ & $38.5(21.3)$ & $44.4(19.6)$ & $46.4(26.0)$ & $48.6(18.1)$ \\
\hline N2 sleep (min) & $212.6(62.1)$ & $219.7(68.6)$ & $212.1(55.6)$ & $215.9(59.7)$ & $195.8(63.1)$ \\
\hline N3 sleep (min) & $71.1(32.4)$ & $72.3(30.7)$ & $79.8(30.8)$ & $57.9(29.0)$ & $61.3(36.5)$ \\
\hline REM sleep (min) & $73.8(26.7)$ & $73.6(29.0)$ & $78.3(24.0)$ & $70.6(27.4)$ & $67.0(26.4)$ \\
\hline Obstructive apnoea index (events/h) & $0.1(0.6)$ & 0 & $0.03(0.1)$ & $0.04(0.1)$ & $0.7(1.4)$ \\
\hline Obstructive hypopnoea index (events/h) & $8.5(8.0)$ & $2.9(1)$ & $6.2(1.7)$ & $11.7(1.9)$ & $20.6(7.1)$ \\
\hline $\begin{array}{l}\text { Obstructive hypopnoeas associated with } \\
\text { microarousals_-index (events/h) }\end{array}$ & $6.3(5.6)$ & $2.1(0.8)$ & $4.7(1.4)$ & $9.4(2.3)$ & $15.5(7.0)$ \\
\hline $4 \%$ oxygen desaturation index (events/h) & $0.8(2.5)$ & $0.13(0.4)$ & $0.44(0.7)$ & $0.38(0.8)$ & $3.44(5.4)$ \\
\hline Oxygen saturation nadir (\%) & $91(6)$ & $92(2)$ & $91(5)$ & $88(8)$ & $88(9)$ \\
\hline Microarousal index (events/h) & $27.1(10.6)$ & $22.1(7.6)$ & $25(8.5)$ & $32.0(8)$ & $37.4(13)$ \\
\hline
\end{tabular}

indices such as obstructive hypopnoea index, and the oxygen desaturation index (table 5), were significantly associated with an increased odds of delivering an SGA infant. While the apnoea index was not significantly associated with the delivery of an SGA baby, the mean apnoea index of the cohort was only 0.1 (table 2). The most frequent respiratory events were obstructive hypopnoeas associated with microarousals, which were significantly associated with an increased odds of delivering an SGA infant (table 5). This finding was specific for respiratory-related arousals in that there was no significant association between spontaneous microarousals and SGA.

Symptoms of snoring and/or witnessed apnoeas were not significantly associated with increased odds of delivering an SGA baby in this cohort (table 5). However, symptoms in the third trimester or new or incident symptoms (from the first to the third trimesters) suggested increased risk for delivering an SGA baby, but just failed to reach statistical significance $(\mathrm{p}=0.1$ and 0.08 , respectively). Neck circumference, a physical measurement that is known to be associated with SDB, was found to be associated with SGA in this cohort (OR 1.25 (95\% CI 1.03 to 1.53 ) per $1 \mathrm{~cm}$ increase, $\mathrm{p}=0.026$ ).

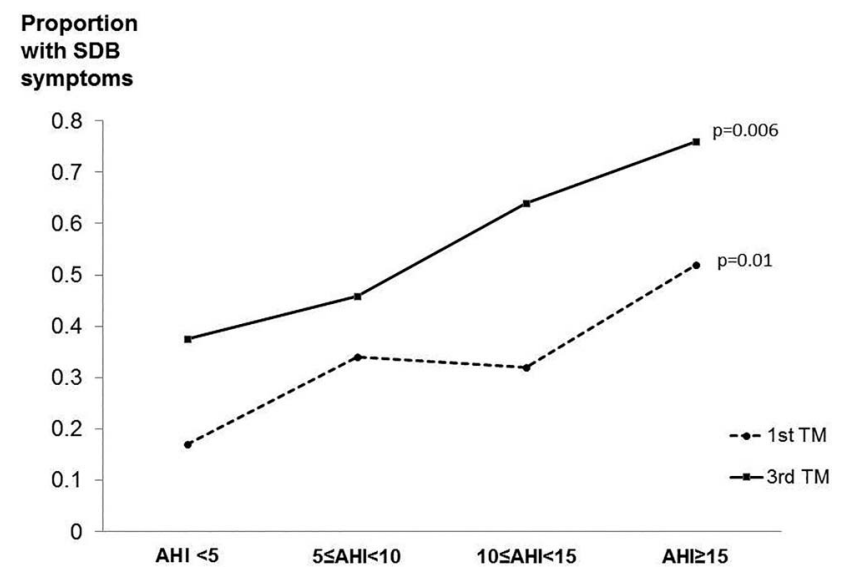

Figure 2 Relationship of sleep-disordered breathing (SDB) symptoms and apnoea-hypopnoea index (AHI). Proportion of participants who reported SDB symptoms in the first trimester (dotted line) versus the third trimester (solid line) according to AHI severity. p Values for trend are shown for the first and third trimesters.
In a subset of our participants who had umbilical artery Doppler ultrasound in the third trimester, customised birth centiles, rather than population-based birth centiles, were significantly associated $(r=-0.43 ; p=0.0005)$ with an increase in the mean resistance index, a measure which has been associated with adverse outcomes. ${ }^{38}$

\section{DISCUSSION}

In this relatively healthy and non-obese cohort, we demonstrated that a PSG-based diagnosis of SDB using various AHI thresholds in the third trimester of pregnancy was associated with a significantly increased odds of delivering an SGA baby (ORs ranging between 2.57 and $3.07 ; \mathrm{p}<0.05$ ). In addition, other PSG-based indices of SDB, including the obstructive hypopnoea index and the oxygen desaturation index, were consistently associated with an increased odds of delivering an SGA infant. Although symptoms of SDB failed to reach statistical significance for an association with the delivery of SGA babies, this may be due to limited power. The definition of SGA in our study was based on customised birth centiles ( $<10$ th centile) and was adjusted for important confounders such as maternal prepregnancy BMI, parity, ethnicity, fetal sex and gestational age

Table 3 Sensitivity and specificity of self-reported snoring and/or witnessed apnoeas versus PSG-based diagnosis of sleep-disordered breathing

\begin{tabular}{|c|c|c|c|}
\hline $\begin{array}{l}\text { Diagnostic } \\
\text { test result } \\
\text { parameter } \\
(\%, 95 \% \mathrm{Cl})\end{array}$ & $\mathrm{AHI} \geq 5$ & $\mathrm{AHI} \geq 10$ & $A H I \geq 15$ \\
\hline \multicolumn{4}{|l|}{ First trimester } \\
\hline Sensitivity & 37.7 (28.8 to 47.3 ) & 41.7 (27.6 to 56.8$)$ & 52.0 (31.3 to 72.2 ) \\
\hline Specificity & 83.0 (71.0 to 91.6$)$ & 73.6 (65.0 to 81.0$)$ & 73.0 (65.1 to 80.0$)$ \\
\hline \multicolumn{4}{|l|}{ Third trimester } \\
\hline Sensitivity & 57.1 (47.4 to 66.4$)$ & 70.6 (56.2 to 82.5 ) & 76.9 (56.3 to 91.0$)$ \\
\hline Specificity & 63.2 (49.3 to 75.5$)$ & 58.5 (49.0 to 67.5$)$ & 54.5 (46.0 to 62.9 ) \\
\hline
\end{tabular}

Pamidi S, et al. Thorax 2016;71:719-725. doi:10.1136/thoraxjnl-2015-208038 
Table 4 Infant birthweight outcome data of pregnancy cohort according to the AHI stratum

\begin{tabular}{|c|c|c|c|c|c|c|}
\hline & Complete cohort $(n=234)$ & $A H I<5(n=77)$ & $5 \leq \mathrm{AHI}<10(\mathrm{n}=84)$ & $10 \leq A H I<15(n=33)$ & $A H I \geq 15(n=36)$ & $p$ Value \\
\hline Gestational age at delivery (weeks) & $39.2(1.2)$ & $39.3(1.3)$ & $39.4(1.1)$ & $39.4(1.1)$ & $39.0(1.3)$ & 0.2 \\
\hline Preterm birth & $16(8 \%)$ & $6(8 \%)$ & $4(5 \%)$ & $1(3 \%)$ & $5(14 \%)$ & 0.3 \\
\hline Infant birth weight, $g$ & 3300 (394) & 3330 (346) & $3335(401)$ & 3179 (357) & $3290(425)$ & 0.2 \\
\hline Infant sex, female & $115(50 \%)$ & 37 (49\%) & $38(45 \%)$ & $17(53 \%)$ & $19(56 \%)$ & 0.2 \\
\hline
\end{tabular}

Values are means (SD) or numbers (\%).

p Values for trend are shown using the Cochran-Armitage trend test $(36,37)$. Preterm birth was defined as live births $<37$ weeks gestational age.

AHI, apnoea-hypopnoea index.

at birth. These findings may have important implications for the perinatal management of maternal SDB.

We also demonstrated that in a subset of participants who completed the PSQI in the first and third trimesters $(n=182)$, symptoms of SDB increased in parallel with AHI severity. However, symptoms of SDB were of overall poor sensitivity and specificity for predicting PSG-based diagnosis of SDB and were not predictive of delivering an SGA baby. The few previous studies examining the link between maternal SDB and the delivery of SGA babies as a primary outcome have yielded mixed results. However, the majority of studies used symptom-based assessments of SDB, ${ }^{15} 16353940$ and the timing of ascertainment of symptoms during pregnancy and exact symptom definition of SDB varied significantly. Of the two studies that used PSG-based diagnoses of SDB, one was a retrospective cohort study $^{23}$ that showed no association and the other, a large database study $^{22}$ demonstrated a positive association. One recent small study by Fung et al used PSG to study 51 pregnant women and identified 14 with sleep apnoea, which was associated with delivering babies with impaired fetal growth. ${ }^{41}$ These authors also used customised birthweight centiles to define SGA, but found a significant association between sleep apnoea and impaired fetal growth (defined as either a birth weight $<10$ th centile or fall in customised centile $>33 \%$ between 32 weeks and term) but not SGA defined by birth

\section{Proportion \\ of SGA births}
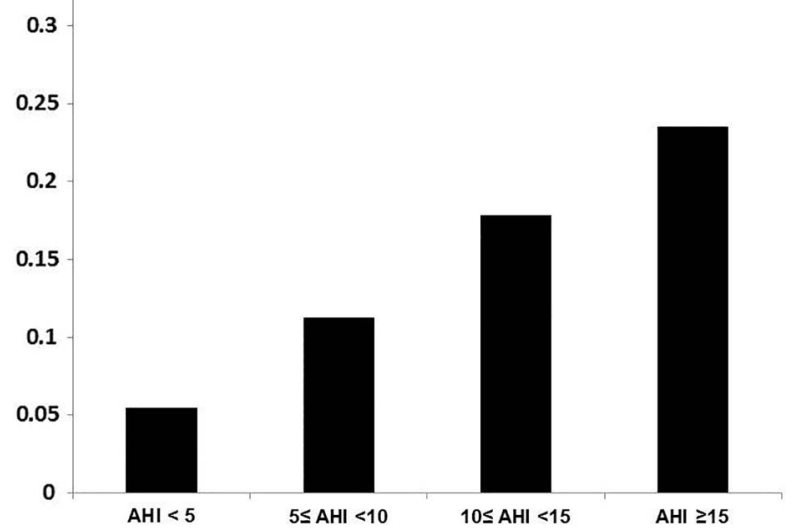

Figure 3 Relationship between severity of maternal sleep-disordered breathing and delivery of small for gestational age (SGA) infants. Proportion of infants born SGA to mothers with increasing severity of sleep-disordered breathing as represented by apnoea-hypopnoea index (AHI) categories. SGA was defined as $<10$ th centile according to customised growth charts that adjusted for maternal parity, height, prepregnancy weight, ethnicity, gestational age at delivery, infant sex and infant birth weight. $p=0.01$ ( $p$ for trend using mean AHI of each category). weight $<10$ th centile alone. ${ }^{41}$ It is likely that the smaller sample size in the study by Fung et al contributed to failure to detect a significant relationship between SGA and SDB.

The use of customised birthweight centiles to define SGA, rather than population-based birth centiles, has been shown to lead to fewer misclassifications of SGA infants. Customised birthweight centiles were initially proposed by Gardosi et al 2829 to account for maternal characteristics that influence fetal growth, and account for maternal height, prepregnancy weight, parity, ethnicity and fetal sex. Due to the adjustments for these contributors to fetal growth, customised centiles better reflect infants who are SGA due to pathological IUGR, rather than infants who are constitutionally small but have reached their growth potential. ${ }^{28}{ }^{29}$ Customised growth centiles have been demonstrated to be superior to conventionally used birthweight centiles that are adjusted only for infant sex and gestational age in predicting adverse maternal and fetal outcomes. ${ }^{42}$ The association between umbilical artery Doppler ultrasound and customised birth centiles that was demonstrated in a subset of our participants further suggests that SDB affects fetal growth on the basis of placental dysfunction.

In our study, most SDB in the third trimester was mild and characterised by obstructive hypopnoeas associated with microarousals, rather than by oxygen desaturations or obstructive apnoeas, as has been demonstrated previously in pregnancy. ${ }^{43} 44$ Thus, it is not surprising that obstructive hypopnoeas, rather than obstructive apnoeas, were associated with SGA babies since very few obstructive apnoeas were observed. Since the scoring criteria and AHI thresholds are still not well established in pregnancy, we presented our data using various AHI thresholds and PSG indices and used sensitive (Chicago) scoring criteria to avoid misclassification of SDB.

Strengths of our study included its prospective nature, and the relatively large number of participants studied late in the third trimester with complete PSG that included EEG for sleep staging, which is more accurate for AHI determination. Moreover, symptoms of SDB (snoring and/or witnessed apnoeas) were ascertained both in the first and third trimesters of pregnancy to assess the relationship with PSG measures, since this has not been well examined in the literature. We also minimised misclassification of SGA by using customised birth centiles adjusted for important maternal and fetal characteristics that can influence birth weight. We used extensive and broad definitions of SDB using various AHI cut-offs and also examined all indices of PSG-based diagnosis of SDB to ensure our associations were robust and consistent.

Our study also had important limitations. First, we only had 27 cases of SGA (12\%) in our cohort, which limits statistical power and ability to control for additional potential confounding variables, such as socioeconomic status and education. On the other hand, despite the small number of cases, we were able to find statistically significant relationships across several 
Figure 4 Mean indices of maternal sleep-disordered breathing in non-SGA (solid bars) and SGA (shaded bars) infants. Error bars represent SEM. SGA, small for gestational age.
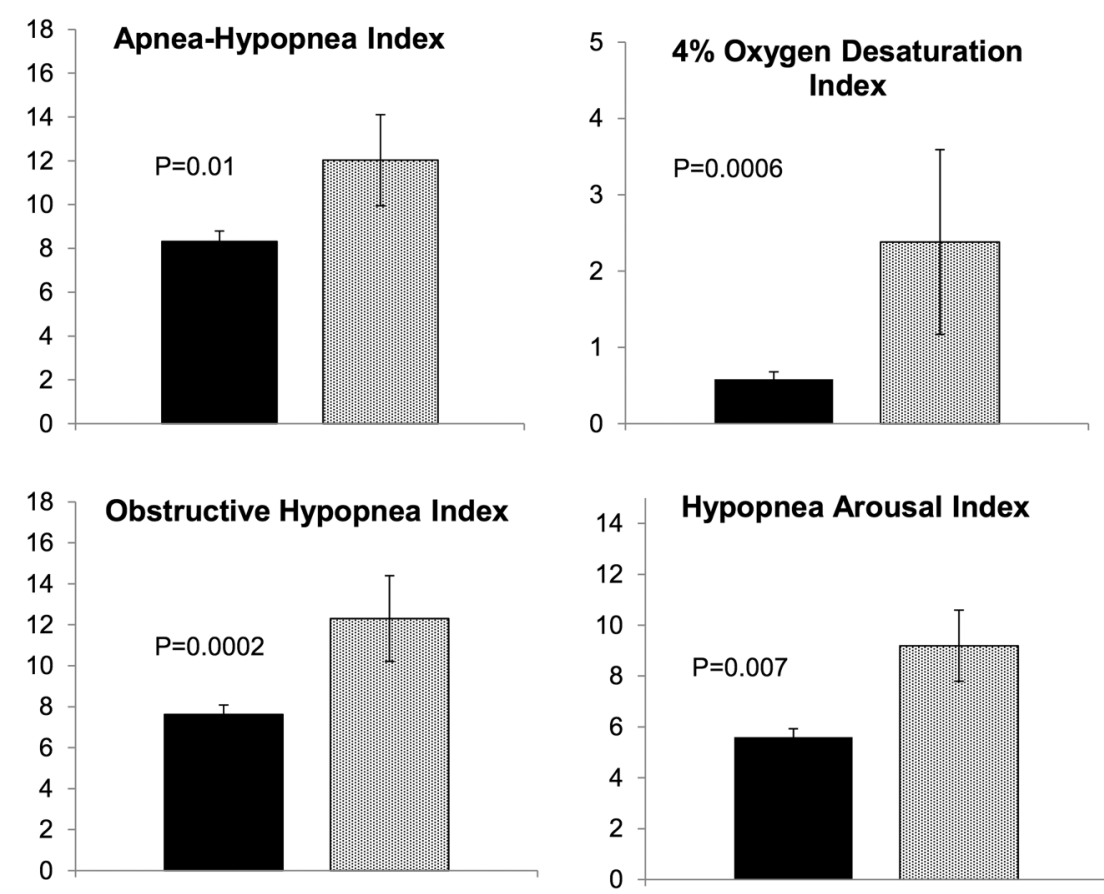

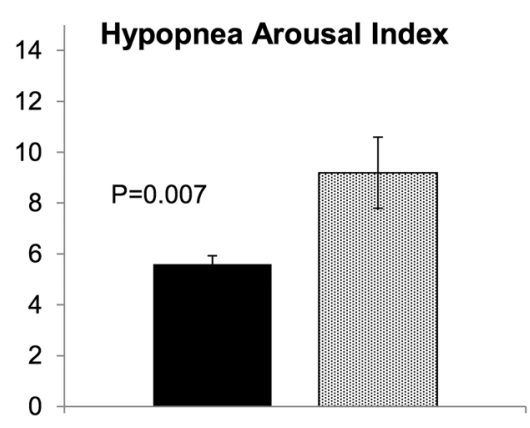

PSG-based measurements and indices for SDB, suggesting that our findings were consistent. Another limitation is that 182 participants completed the symptom questionnaires (PSQI), and thus we were left with missing data. Despite the limited sensitivity and specificity of symptoms (table 3), we did see some potential associations of symptoms in the third trimester and new incident symptoms with delivery of SGA infants. Thus, the smaller sample size from non-response resulted in more limited power with respect to finding a significant relationship between symptoms and delivery of SGA babies. We also used different thresholds to define symptom-based SDB (ie, snoring and/or

Table 5 ORs of maternal SDB for the delivery of an SGA infant versus a non-SGA infant

\begin{tabular}{|c|c|c|}
\hline Predictor & OR $(95 \% \mathrm{Cl})$ & p Value \\
\hline \multicolumn{3}{|l|}{ SDB symptoms } \\
\hline $\begin{array}{l}\text { Any snoring or witnessed apnoeas in the } \\
\text { first trimester (ref: no snoring) }\end{array}$ & $1.10(0.35$ to 3.38$)$ & 0.87 \\
\hline $\begin{array}{l}\text { Any snoring or witnessed apnoeas in the } \\
\text { third trimester (ref: no snoring) }\end{array}$ & $2.36(0.85$ to 6.54$)$ & 0.10 \\
\hline $\begin{array}{l}\text { New or incident snoring by the third } \\
\text { trimester (ref: no new snoring by } 3 \text { rd } \\
\text { trimester) }\end{array}$ & $3.78(0.84$ to 17.01$)$ & 0.08 \\
\hline \multicolumn{3}{|l|}{ PSG-based measurements of SDB } \\
\hline$A H I \geq 5$ events/h & 3.07 (1.01 to 9.26$)$ & 0.047 \\
\hline $\mathrm{AHI} \geq 10$ events $/ \mathrm{h}$ & $2.65(1.15$ to 6.10$)$ & 0.022 \\
\hline $\mathrm{AHI} \geq 15$ events $/ \mathrm{h}$ & 2.57 (1.02 to 6.48$)$ & 0.045 \\
\hline AHI, 10 events/h & 1.48 (1.01 to 2.18$)$ & 0.043 \\
\hline Obstructive apnoea index, 1 event/h & 1.18 (0.69 to 2.01$)$ & 0.55 \\
\hline Obstructive hypopnoea index, 10 events/h & 1.56 (1.04 to 2.34$)$ & 0.03 \\
\hline Hypopnoea-arousal index, 10 events/h & 2.07 (1.15 to 3.75$)$ & 0.016 \\
\hline $4 \%$ oxygen desaturation index, 1 event/h & $1.17(1.03$ to 1.34$)$ & 0.016 \\
\hline \multicolumn{3}{|c|}{$\begin{array}{l}\text { OR for continuous variables indicate the change in odds for an increase in specified } \\
\text { number. The ORs represent the risk of delivery of an SGA baby as calculated by } \\
\text { customised birthweight centile data (described in Methods) and have been adjusted } \\
\text { for maternal prepregnancy BMI, parity, ethnicity, infant sex and gestational age. } \\
\text { AHI, apnoea-hypopnoea index; BMI, body mass index; PSG, polysomnography; SDB, } \\
\text { sleep-disordered breathing; SGA, small for gestational age. }\end{array}$} \\
\hline
\end{tabular}

witnessed apnoeas at any frequency, symptoms $>1 \times /$ week, or $\geq 3 \times /$ week), and there was no significant change in the results. Furthermore, since we did not have a PSG during the first trimester, we could not assess whether objective first trimester PSG findings impact the risk of delivering an SGA baby. We also cannot draw conclusions on whether it is new or incident SDB during pregnancy versus pre-existing SDB that is a risk factor for delivering an SGA baby.

We had 15 participants who were missing some of the maternal characteristics required for accurate assessment of SGA customisation. However, since only two participants were missing birthweight data, we performed an additional sensitivity analysis and imputed the mean value from the cohort for the missing maternal parameter and recalculated the customised birth centile. This did not change our results significantly. Finally, our customised centiles and coefficients for the various maternal characteristics were based on USA-based population data, as there has not yet been a Canadian standard developed for calculating customised centiles. However, the distribution of the ethnicity of our cohort fits best with the US data set (ie, coefficient for the Latin American population available) rather than the European or Australian coefficients.

Biological plausibility for gestational exposure to SDB and low birth weight has already been demonstrated in rat models of gestational intermittent hypoxia where asymmetrical growth restriction in addition to overall reduced birth weights have been demonstrated. ${ }^{13}{ }^{14}$ Our clinical data are consistent with these findings and also demonstrate that objective PSG diagnosis of SDB, rather than symptom-based assessments, are linked with an increased risk of delivering infants that are SGA, suggesting that the use of objective-based measurements of maternal SDB is important for future studies. Our results highlight that SDB, although prevalent in this cohort, is overall mild in severity, similar to what other studies in pregnancy and SDB have observed. ${ }^{45}$ Despite this, the mild degree of SDB is associated with adverse fetal outcomes. Thus, the use of routine ambulatory sleep studies, which largely depend on oxygen desaturation events rather than arousals, may not detect the large majority of $\mathrm{SDB}$ in pregnancy. However, future work is still necessary to 
compare the accuracy and practical issues in performing ambulatory versus in-laboratory PSG in pregnancy. Finally, to further understand the causal nature of the relationship between maternal SDB and the delivery of SGA infants, interventional trials involving treatment of maternal SDB with CPAP will be needed.

Acknowledgements The authors thank the sleep technologists, Chris Brookes, Marcel Severe and Thao linh Dang for their assistance in setting up the sleep recordings. The authors also thank Audree Janelle-Montcalm, Romy-Leigh McMaster and Isabelle Krauss for their assistance with data management from the parent IRNPQEO cohort. The authors thank Victoria Mery for helping review sleep studies. The authors also thank the research participants in this study.

Contributors Conception and design: SP, IM and RJK. Acquisition of data: SP, IM, $A O, L L, F S, W F$ and RJK. Analysis and interpretation of data: SP, IM, GS, AB, RJK. Drafting, revising and critical revision for intellectual content and final approval of the version to be published: $S P, I M, L L, A O, F S, W F, F A, E B, R G, G S, A B, K S, R J K$. All authors are in agreement to be accountable for the work presented in the manuscript.

Funding Supported by: IRNPQEO: Canadian Institutes of Health Research (CIHR) (Grant Ref. No. CRI 88413); SGA substudy: CIHR Sleep and Circadian Rhythm Grant Ref. No.104728, in partnership with Canadian Lung Association and the Fonds de recherche du Québec—Santé (FRQS).

Competing interests None declared.

Ethics approval McGill University Health Centre, Le Centre hospitalier universitaire Sainte-Justine, Montreal, Quebec, Canada.

Provenance and peer review Not commissioned; externally peer reviewed.

\section{REFERENCES}

1 Breeze AC, Lees CC. Prediction and perinatal outcomes of fetal growth restriction. Semin Fetal Neonatal Med 2007;12:383-97.

2 Barker DJ. The long-term outcome of retarded fetal growth. Clin Obstet Gynecol 1997:40:853-63.

3 McIntire DD, Bloom SL, Casey BM, et al. Birth weight in relation to morbidity and mortality among newborn infants. N Engl J Med 1999:340:1234-8.

4 Lausman A, McCarthy FP, Walker M, et al. Screening, diagnosis, and management of intrauterine growth restriction. J Obstet Gynaecol Can 2012;34:17-28.

5 Arslan M, Yazici G, Erdem A, et al. Endothelin 1 and leptin in the pathophysiology of intrauterine growth restriction. Int I Gynaecol Obstet 2004;84:120-6.

6 Mifsud W, Sebire NJ. Placental pathology in early-onset and late-onset fetal growth restriction. Fetal Diagn Ther 2014;36:117-28.

7 Baguet JP, Barone-Rochette G, Tamisier R, et al. Mechanisms of cardiac dysfunction in obstructive sleep apnea. Nat Rev Cardiol 2012;9:679-88.

8 Arnaud C, Dematteis M, Pepin JL, et al. Obstructive sleep apnea, immuno-inflammation, and atherosclerosis. Semin Immunopathol 2009;31:113-25.

9 Lattimore JD, Celermajer DS, Wilcox I. Obstructive sleep apnea and cardiovascular disease. J Am Coll Cardiol 2003;41:1429-37.

10 Monahan K, Redline S. Role of obstructive sleep apnea in cardiovascular disease. Curr Opin Cardiol 2011;26:541-7.

11 Pepin JL, Tamisier R, Levy P. Obstructive sleep apnoea and metabolic syndrome: put CPAP efficacy in a more realistic perspective. Thorax2012:67:1025-7.

12 Pamidi S, Tasali E. Obstructive sleep apnea and type 2 diabetes: is there a link? Front Neurol 2012:3:126.

13 Gozal D, Reeves SR, Row BW, et al. Respiratory effects of gestational intermittent hypoxia in the developing rat. Am J Respir Crit Care Med 2003;167:1540-7.

14 Iqbal W, Ciriello J. Effect of maternal chronic intermittent hypoxia during gestation on offspring growth in the rat. Am J Obstet Gynecol 2013;209:564.e1-9.

15 Bourjeily G, Raker CA, Chalhoub M, et al. Pregnancy and fetal outcomes of symptoms of sleep-disordered breathing. Eur Respir J 2010;36:849-55.

16 Franklin KA, Holmgren PA, Jonsson F, et al. Snoring, pregnancy-induced hypertension, and growth retardation of the fetus. Chest 2000;117:137-41.

17 Loube DI, Poceta JS, Morales MC, et al. Self-reported snoring in pregnancy. Association with fetal outcome. Chest 1996;109:885-9.

18 Redolfi S, Yumino D, Ruttanaumpawan P, et al. Relationship between overnight rostral fluid shift and Obstructive Sleep Apnea in nonobese men. Am I Respir Crit Care Med 2009;179:241-6.
19 Izci-Balserak B, Pien GW. Sleep-disordered breathing and pregnancy: potential mechanisms and evidence for maternal and fetal morbidity. Curr Opin Pulm Med 2010;16:574-82.

20 Edwards N, Middleton PG, Blyton DM, et al. Sleep disordered breathing and pregnancy. Thorax 2002;57:555-8.

21 Pamidi S, Pinto LM, Marc I, et al. Maternal sleep-disordered breathing and adverse pregnancy outcomes: a systematic review and metaanalysis. Am J Obstet Gynecol 2014;210:52.e1-52.e14

22 Chen $\mathrm{YH}$, Kang JH, Lin CC, et al. Obstructive sleep apnea and the risk of adverse pregnancy outcomes. Am J Obstet Gynecol 2012;206:136.e1-5.

23 Louis JM, Auckley D, Sokol RJ, et al. Maternal and neonatal morbidities associated with obstructive sleep apnea complicating pregnancy. Am J Obstet Gynecol 2010:202:261.e1-5

24 Pamidi S, Marc I, Lavigne L, et al. The association between maternal sleep apnea and small for gestational age infants. Presented at the American Thoracic Society Conference, Philadelphia, PA, 2013:A5942-A42.

25 Pamidi S, Marc I, Lavigne L, et al. Risk of delivering low birth weight infants in symptom- and PSG-based assessment of maternal sleep-disordered breathing. Presented at the American Thoracic Society Conference, Denver, CO, 2015: A3937-A37.

26 Hadlock FP, Harrist RB, Sharman RS, et al. Estimation of fetal weight with the use of head, body, and femur measurements - a prospective study. Am J Obstet Gynecol 1985;151:333-7.

27 Edwards N, Blyton DM, Hennessy A, et al. Severity of sleep-disordered breathing improves following parturition. Sleep 2005;28:737-41.

28 Gardosi J, Chang A, Kalyan B, et al. Customised antenatal growth charts. Lancet 1992;339:283-7.

29 Gardosi J, Mongelli M, Wilcox M, et al. An adjustable fetal weight standard. Ultrasound Obstet Gynecol 1995;6:168-74.

30 Gardosi J, Francis A. GROW centile calculator v5.7.5.4. Secondary GROW centile calculator v5.7.5.4. http://www.gestation.net (accessed May 2015).

31 Berry RB, Brooks R, Gamaldo CE, et al. The AASM manual for the scoring of sleep and associated events: rules, terminology and technical specifications. Version 2.1. Secondary The AASM manual for the scoring of sleep and associated events: rules, terminology and technical specifications. Version 2.1. 2013. http://www.aasmnet.org

32 Sleep-related breathing disorders in adults: recommendations for syndrome definition and measurement techniques in clinical research. The Report of an American Academy of Sleep Medicine Task Force. Sleep 1999;22:667-89.

33 Ruehland WR, Rochford PD, O'Donoghue FJ, et al. The new AASM criteria for scoring hypopneas: impact on the apnea hypopnea index. Sleep 2009;32:150-7.

34 Buysse DJ, Reynolds CF III, Monk TH, et al. The Pittsburgh Sleep Quality Index: a new instrument for psychiatric practice and research. Psychiatry Res 1989;28:193-213.

35 Micheli K, Komninos I, Bagkeris E, et al. Sleep patterns in late pregnancy and risk of preterm birth and fetal growth restriction. Epidemiology 2011;22:738-44.

36 Armitage P. Tests for linear trends in proportions and frequencies. Biometrics 1955;11:375-86.

37 Cochran WG. Some methods of strengthening the common chi-squared tests. Biometrics 1954;11:417-51.

38 Albaiges G, Missfelder-Lobos H, Parra M, et al. Comparison of color Doppler uterine artery indices in a population at high risk for adverse outcome at 24 weeks' gestation. Ultrasound Obstet Gynecol 2003;21:170-3.

39 Perez-Chada D, Videla AJ, O'Flaherty ME, et al. Snoring, witnessed sleep apnoeas and pregnancy-induced hypertension. Acta Obstet Gynecol Scand 2007:86:788-92.

40 Yin TT, Williams N, Burton C, et al. Hypertension, fetal growth restriction and obstructive sleep apnoea in pregnancy. Eur I Obstet Gynecol Reprod Biol 2008;141:35-8.

41 Fung AM, Wilson DL, Lappas M, et al. Effects of maternal obstructive sleep apnoea on fetal growth: a prospective cohort study. PLoS ONE 2013;8:e68057.

42 Gardosi J, Francis A. Adverse pregnancy outcome and association with small for gestational age birthweight by customized and population-based percentiles. Am J Obstet Gynecol 2009;201:28.e1-8.

43 Pien GW, Fife D, Pack Al, et al. Changes in symptoms of sleep-disordered breathing during pregnancy. Sleep 2005;28:1299-305.

44 Connolly G, Razak AR, Hayanga A, et al. Inspiratory flow limitation during sleep in pre-eclampsia: comparison with normal pregnant and nonpregnant women. Eur Respir J 2001;18:672-6.

45 Pien GW, Pack Al, Jackson N, et al. Risk factors for sleep-disordered breathing in pregnancy. Thorax2014;69:371-7. 